\title{
Impulse-driven Surface Breakdown Data: A Weibull Statistical Analysis
}

\author{
Mark P. Wilson, Member, IEEE, Martin J. Given, Senior Member, IEEE, Igor V. Timoshkin, Member, IEEE, \\ Scott J. MacGregor, Member, IEEE, Tao Wang, Mark A. Sinclair, Member, IEEE, \\ Kenneth J. Thomas, Senior Member, IEEE, and Jane M. Lehr, Fellow, IEEE
}

\begin{abstract}
Surface breakdown of oil-immersed solids chosen to insulate high-voltage, pulsed-power systems is a problem that can lead to catastrophic failure. Statistical analysis of the breakdown voltages, or times, associated with such liquid-solid interfaces can reveal useful information to aid system designers in the selection of solid materials. Described in this paper are the results of a Weibull statistical analysis, applied to both breakdown-voltage data and time-to-breakdown data generated in gaps consisting of five different solid polymers immersed in mineral oil. Values of the location parameter $\gamma$ provide an estimate of the applied voltage below which breakdown will not occur, and under uniform-field conditions, $\gamma$ varied from $192 \mathrm{kV}$ for polypropylene (PP) to zero for ultrahigh-molecular-weight polyethylene (UHMWPE). Longer times to breakdown were measured for UHMWPE when compared with the other materials. However, high values of the shape parameter $\beta$ reported in the present paper suggest greater sensitivity to an increase in applied voltage-that is, the probability of breakdown increases more sharply with increasing applied voltage for UHMWPE compared to the other materials. Analyzing peak-applied-voltage data, only PP consistently reflected a low value of $\boldsymbol{\beta}$ across the different sets of test conditions. In general, longer mean times to breakdown were found for solid materials with permittivity more closely matched to that of the surrounding mineral oil.
\end{abstract}

Index Terms-Breakdown voltage, dielectric breakdown, flashover, pulse power systems, Weibull distribution.

\section{INTRODUCTION}

$\mathbf{T}$ HE INTRODUCTION of solid dielectrics into liquid insulation located between two electrical conductors is often necessary to provide mechanical support in subsystems of high-voltage, pulsed-power machines, and it is necessary to understand the insulation behavior of such liquid-solid gaps. Information on the variation in breakdown voltage/time data with an increasing number of breakdown events for liquid-solid gaps subjected to overvoltages, and for breakdown initiation measurements, are reported in [1]. Five different solid materials were tested, namely polypropylene (PP);

Manuscript received October 14, 2011; revised ; accepted December 16, 2011. This work was supported by AWE Aldermaston.

M. P. Wilson, M. J. Given, I. V. Timoshkin, S. J. MacGregor, and T. Wang are with the Department Electronic \& Electrical Engineering, University of Strathclyde, G1 1XW Glasgow, U.K. (e-mail: m.wilson@eee.strath.ac.uk; m.given@eee.strath.ac.uk; igor.timoshkin@eee.strath.ac.uk; s.macgregor@ eee.strath.ac.uk; tao.wang@eee.strath.ac.uk).

M. A. Sinclair, and K. J. Thomas are with the Pulsed-Power Group, AWE Aldermaston, RG7 4PR Reading, U.K. (e-mail: mark.sinclair@awe.co.uk; ken.thomas@awe.co.uk).

J. M. Lehr is with the Sandia National Laboratories, Albuquerque, NM 87185 USA (e-mail: jmlehr@sandia.gov).

Digital Object Identifier 10.1109/TPS.2011.2181172 low-density polyethylene (LDPE); ultrahigh-molecular-weight polyethylene (UHMWPE); Rexolite (cross-linked polystyrene); and Torlon (polyamide-imide). The applied voltage wave had a front time of $100 \mathrm{~ns}$ and a time to half-value of $600 \mathrm{~ns}$, measured in accordance with IEC 60060-1 [2]. The impulses were of negative polarity and magnitude up to $450 \mathrm{kV}$. In this paper, the peak applied field necessary to initiate surface breakdown events was found to decrease with increasing relative permittivity of the solid and corresponding increasing permittivity mismatch between the solid and the surrounding mineral oil. This finding is in agreement with those of Taylor [3] and Krins et al. [4], both of which studies found that higher flashover voltages were yielded by matching the permittivity of the parallel solid surface to that of the oil in which it was immersed.

In the case of self-restoring insulation, for example, mineral oil without a solid spacer, lightning-impulse withstand voltage test methods presently used in the power industry include the multiple-level method and the up-and-down method [2]. These test procedures involve evaluation of the voltage where the probability of the occurrence of a disruptive discharge is $10 \%$ $\left(U_{10}\right)$, and the withstand test is considered to be passed if $U_{10}$ is "not less than the specified impulse withstand voltage."

The present paper is focused on the statistical analysis of the breakdown initiation results reported for both uniformand nonuniform-field measurements in [1]. The breakdown initiation test procedure used in [1] was similar to the step-up methods described by Hirose [5] as being suitable for the estimation of the breakdown voltage of non-self-restoring electrical insulation. The initial voltage level applied to the insulation system is sufficiently low in order that no breakdown event will occur. The applied voltage is then increased incrementally. If electrical breakdown of the insulation system occurs, resulting in the generation of experimental breakdown voltage/time data, then the test method can be termed the new step-up method, rather than the (conventional) step-up method, where the insulation system is not broken [6]. The applied voltage was increased in increments of $\sim 20 \mathrm{kV}$ in [1], resulting in eventual breakdown of the liquid-solid interface in all cases.

A Weibull distribution was utilized for the following analysis, where breakdown is assumed to be caused by a weak point in the system [7]. The Weibull distribution has been reported as appropriate for modeling voltages associated with breakdown of insulation systems [8] and can be utilized to estimate the voltage, and hence the electric field, associated with low probability of breakdown for a particular engineering design. 
The average streamer propagation velocities implied from time-to-breakdown data are also presented and discussed. According to the classification presented in [9], streamers were found to propagate in the third $(10-20 \mathrm{~km} / \mathrm{s})$ and fourth $(100 \mathrm{~km} / \mathrm{s})$ modes in nonuniform fields, and in the second $(1-5 \mathrm{~km} / \mathrm{s})$ and third modes in uniform fields. The upper streamer velocity in the present study was over $100 \mathrm{~km} / \mathrm{s}$.

\section{EXPERIMENTAL ARRANGEMENT}

The experimental system utilized to generate the breakdown voltage/time data comprised a high-voltage impulse generator, an adaptable test cell, and voltage monitoring diagnostics. The impulse generator is a ten-stage, air-insulated, inverting Marx generator, capable of producing output voltages in the region of $500 \mathrm{kV}$. The erected capacitance of the generator is 8 $\mathrm{nF}$. The storage capacitors are charged by a positive-polarity, Glassman high-voltage de supply. A $200 \Omega$ aqueous copper sulphate $\left(\mathrm{CuSO}_{4}\right)$ wave-tail resistor and a $600 \Omega \mathrm{CuSO}_{4}$ wavefront resistor were utilized as wave-shaping components. The impulse front time was $\sim 100 \mathrm{~ns}$, with a time to half-value of $\sim 600 \mathrm{~ns}$.

The test cell consists of a pair of aluminium electrodes, between which the solid polymer samples are inserted for testing. The interelectrode gap is readily adjustable by varying the position of the earth electrode, which consists of a 50-mmdiameter plane. A further three interchangeable electrodes, also $50 \mathrm{~mm}$ in diameter, are used as high-voltage electrodes. Two of these electrodes (types A and B) provide different nonuniformfield distributions, with features designed to initiate discharges in close proximity to the sample surface: for the type A electrode, a 3-mm-long, 1-mm-diameter, tungsten pin protrudes perpendicular to the surface, at a radial distance of $12.5 \mathrm{~mm}$ from the electrode center; while for the type B electrode, a 25-mm-diameter, aluminium collar protrudes perpendicular to the surface at the center of the plane. The third type (type C) has no modifications and is used to provide a plane-parallel configuration. For testing, the cell is immersed in $\sim 20 \mathrm{~L}$ of EOS Ltd. L10B reclaimed insulating oil.

Three different types of solid dielectric sample (types I, II, and III) were machined to match the geometries of the different high-voltage electrodes, each type being based on a cylinder of diameter $25 \mathrm{~mm}$. A sketch of the various combinations of electrode geometry and sample (AI, BIII, and CIII) used in the present study is shown in Fig. 1. A 3-mm-long, 1-mmwide recess is cut into the curved surface at one end of the type I samples, to accommodate the pin from high-voltage electrode type A. The type II samples have a 3-mm-long section ("shoulder") machined to a reduced diameter of $24 \mathrm{~mm}$ at the end to be in contact with the high-voltage electrode, allowing them to be used with both type A and type B electrodes. The type III samples have no modifications and can be used with the type $\mathrm{B}$ and type $\mathrm{C}$ high-voltage electrodes.

Prior to treatment, all solid samples were rinsed in warm water with a little detergent to remove any surface grease and then rinsed in isopropyl alcohol and dried in an oven at $50^{\circ} \mathrm{C}$ for $1 \mathrm{~h}$ to remove surface moisture. Powder-free latex gloves

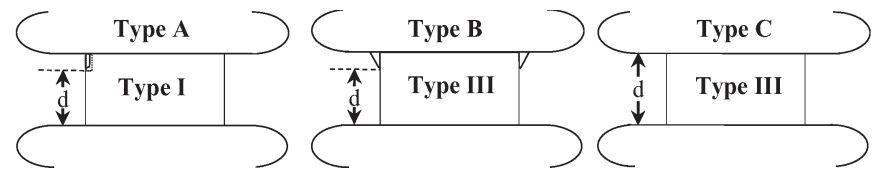

Fig. 1. Sketch of the tested electrode/sample geometries (AI, BIII, and CIII).

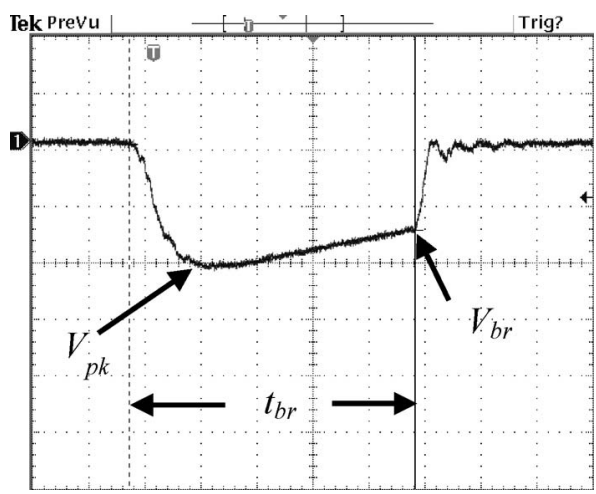

Fig. 2. Voltage waveform illustrating the different parameters recorded for statistical analysis. Vertical scale: $100 \mathrm{kV} /$ division; horizontal scale: 100 ns/division.

were worn throughout the pretreatment process to prevent recontamination of the sample surfaces.

The impulse voltage waveforms were monitored using a Tektronix P6015A probe connected to a tap-off point on a Samtech Ltd. DE(LRP)- $02 \mathrm{CuSO}_{4}$ voltage divider $(2 \mathrm{k} \Omega$ input impedance), giving a measurement system with an overall division ratio of 10 000: 1 . The voltage waveforms were viewed and recorded using a Tektronix TDS3032 digital storage oscilloscope.

\section{STATistical Analysis}

Three parameters were extracted from the experimental voltage waveforms for statistical analysis, as shown in Fig. 2. As breakdown occurred on the impulse falling edge, two voltage levels were recorded, $V_{p k}$ and $V_{b r}$, corresponding to the peak voltage of the breakdown-initiating impulse, and the voltage at the point of breakdown, respectively. The time to breakdown, $t_{b r}$, was also recorded.

\section{A. Peak Applied Voltage}

First, the experimental data recorded for $V_{p k}$ in [1] were subjected to a Weibull statistical analysis, in order to compare the probability of breakdown of the various tested materials at different peak applied voltages. The data were mostly found to be best described by a three-parameter Weibull distribution, where the cumulative probability of failure, $F(V)$, is defined by (1) [7]:

$$
F(V)=1-\exp \left[-\left(\frac{V-\gamma}{\alpha}\right)^{\beta}\right] .
$$

For this section of the analysis, $V$ is the peak applied voltage inducing breakdown $\left(V_{p k}\right)$. As normal, $\alpha$ is the scale parameter, $\beta$ is the shape parameter, and $\gamma$ is the location parameter. The 
TABLE I

Weibull Distribution PARAmeters for $V_{p k}$

*indicates instances where $\alpha$ is the characteristic voltage, where $F(V)=0.632$

\begin{tabular}{|c|c|c|c|c|c|}
\hline Geometry & Material & $\begin{array}{l}\text { Scale } \\
\alpha(\mathrm{kV})\end{array}$ & $\begin{array}{c}\text { Shape } \\
\quad \beta\end{array}$ & $\begin{array}{c}\text { Location } \\
\gamma(\mathrm{kV})\end{array}$ & $\begin{array}{l}\alpha+\gamma \\
(\mathrm{kV})\end{array}$ \\
\hline \multirow{5}{*}{$\begin{array}{l}\begin{array}{l}\text { Pin-plane } \\
(\mathrm{AI})\end{array} \\
d=10 \mathrm{~mm}\end{array}$} & PP & 123 & 1.68 & 190 & 313 \\
\hline & LDPE & 151 & 4.37 & 135 & 286 \\
\hline & UHMWPE & $292^{*}$ & 6.64 & 0 & 292 \\
\hline & Rexolite & $293^{*}$ & 14.5 & 0 & 293 \\
\hline & Torlon & 79 & 2.88 & 120 & 199 \\
\hline \multirow{5}{*}{$\begin{array}{l}\text { Collar-plane } \\
\quad \text { (BIII) } \\
d=10 \mathrm{~mm}\end{array}$} & PP & 115 & 1.56 & 240 & 355 \\
\hline & LDPE & 52 & 1.35 & 240 & 292 \\
\hline & UHMWPE & 69 & 1.17 & 225 & 294 \\
\hline & Rexolite & 79 & 1.52 & 200 & 279 \\
\hline & Torlon & $210^{*}$ & 4.73 & 0 & 210 \\
\hline \multirow{5}{*}{$\begin{array}{c}\text { Plane-parallel } \\
\text { (CIII) } \\
d=4 \mathrm{~mm}\end{array}$} & PP & 66 & 1.75 & 192 & 258 \\
\hline & LDPE & 81 & 1.27 & 160 & 241 \\
\hline & UHMWPE & $206^{*}$ & 4.98 & 0 & 206 \\
\hline & Rexolite & 64 & 2.68 & 165 & 229 \\
\hline & Torlon & 51 & 1.33 & 85 & 136 \\
\hline
\end{tabular}

shape parameter $\beta$ provides a measure of how sensitive the insulation system is to an increase in voltage, while $\gamma(\mathrm{kV})$ provides an estimate of the voltage below which the probability of breakdown is zero [10]. If the location parameter, $\gamma$, is zero, the distribution becomes a two-parameter Weibull distribution; in this case, the scale parameter, $\alpha$, can be considered to be the characteristic breakdown voltage. For a two-parameter distribution, the probability of failure (cumulative percentage of breakdowns), $F(V)$, at a voltage of $\alpha(\mathrm{kV})$ is 0.632 , equivalent to the mean of the normal distribution [10]. When $\gamma$ is nonzero, the characteristic breakdown voltage can be estimated by the sum of $\alpha$ and $\gamma$.

In order to form probability plots to compare the behavior of the different liquid-solid gaps, the cumulative distribution function is converted into a linear form before plotting. When (1) is rearranged into the form of a straight line $(y=m x+c)$, we find (2):

$$
\ln \left[\ln \left(\frac{1}{1-F(V)}\right)\right]=\beta \ln (V-\gamma)-\beta \ln \alpha
$$

where $y$ is equivalent to the left-hand side of the equation, $m$ is equivalent to $\beta, x$ corresponds to $\ln (V-\gamma)$, and $c$ is equivalent to $-\beta \ln (\alpha)$.

The estimated values of $\alpha, \beta$, and $\gamma$ for each of the materials and electrode types are listed in Table I. Analysis of the parameters was performed using Microsoft Excel, with the Analysis ToolPak add-in loaded, as described in [11]. In certain cases, the data were best described by a two-parameter Weibull distribution - in such instances, the value of the location parameter, $\gamma$, is listed as zero in Table I. When $\gamma$ is zero, $\alpha$ represents the characteristic breakdown voltage, where $F(V)=0.632$, and these values are indicated by an asterisk. In order to allow a direct comparison of solid material performance, the values of $\alpha+\gamma$ are included in the final column of Table I, to provide an estimate of the characteristic breakdown voltage in all cases. Comparing the values of $\alpha+\gamma$, it is clear that PP has the highest characteristic breakdown voltage for

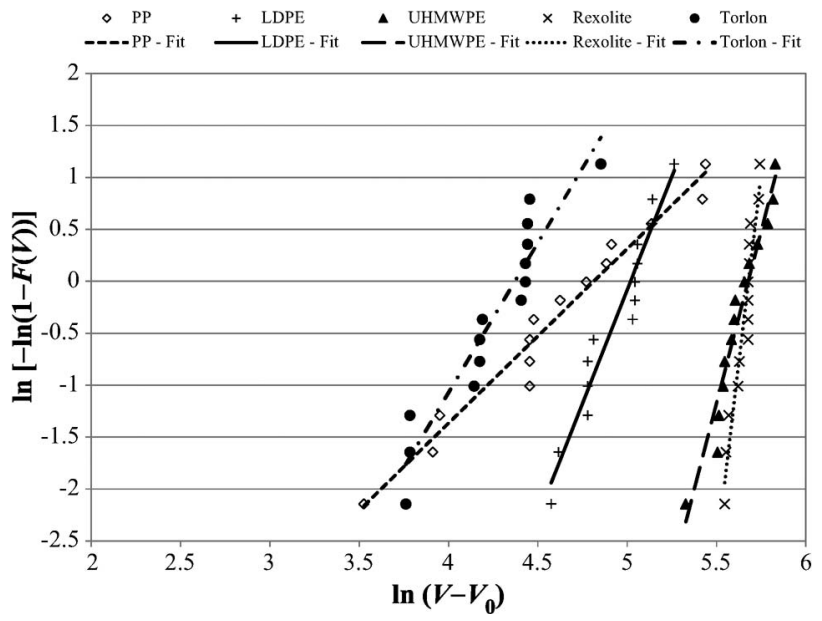

Fig. 3. Weibull plots and curve fits of peak-applied-voltage $\left(V_{p k}\right)$ data for type I (recess) samples tested with high-voltage electrode type A (pin).

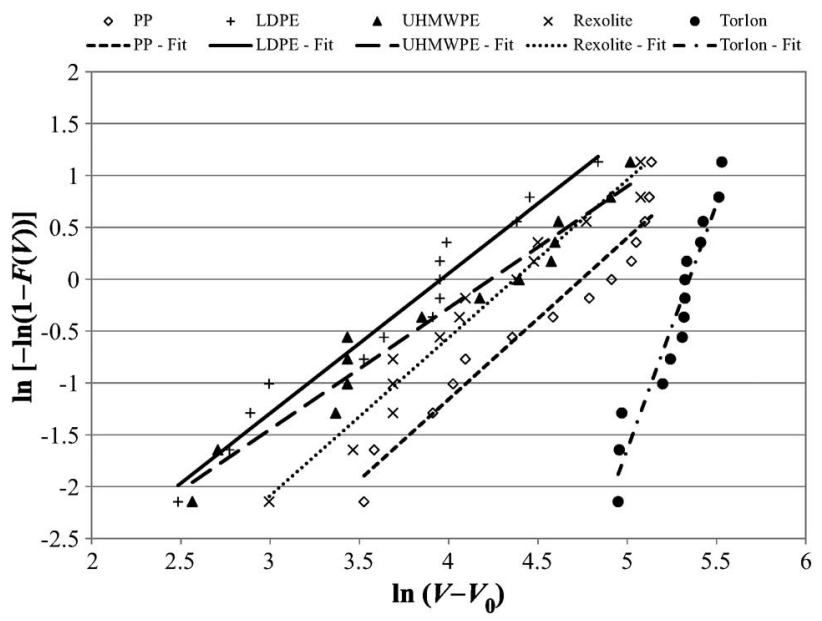

Fig. 4. Weibull plots and curve fits of peak-applied-voltage $\left(V_{p k}\right)$ data for type III (no modifications) samples tested with high-voltage electrode type B (collar).

all geometries. Also, of note is the fact that for plane-parallel electrodes, the characteristic breakdown voltage decreases with increasing $\varepsilon_{r}$ of the solid, and corresponding increasing permittivity mismatch between the solid and the surrounding mineral oil, as discussed further in Section III-C.

Figs. 3-5 show the form of the Weibull plots. The $y$-axis values correspond to the left-hand side of (2), and the $x$ axis values correspond to $\ln (V-\gamma)$. The shape parameter $\beta$ describes the gradient of the straight lines fitted to the data in the probability plots in Figs. 3-5. All values of $\beta$ in Table I are greater than 1 , meaning that the probability of breakdown increases with increasing voltage, as would be expected. The higher the value of $\beta$, the more sensitive the sample is to an increase in voltage- that is, the probability of breakdown increases more sharply with increasing voltage. The extreme case here is Rexolite for pin-plane geometry (AI), where $\beta$ is 14.5, shown by the steep gradient in Fig. 3 .

By analyzing the $\beta$ values in Table $\mathrm{I}$, it is clear that the probability of breakdown is less sensitive to increasing applied voltage for the type B (collar) electrode than for the type A 


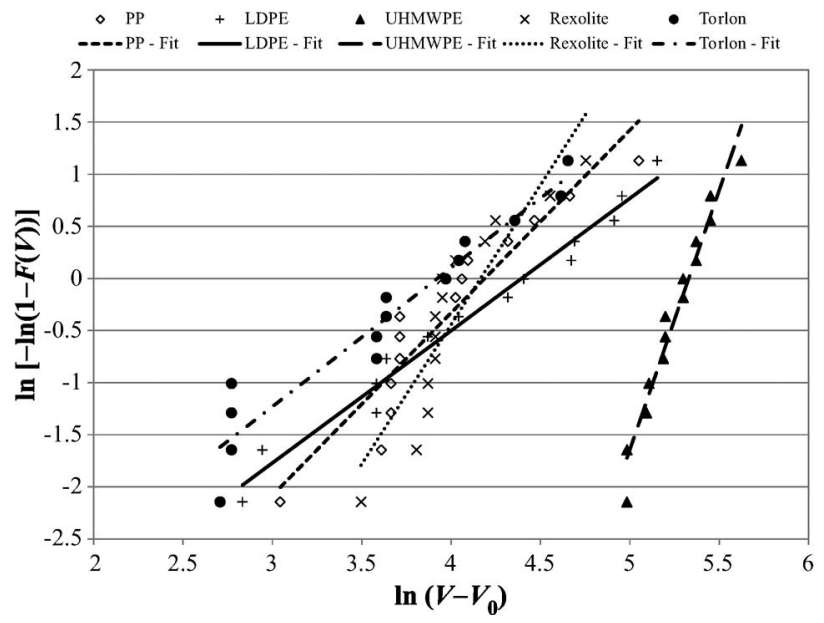

Fig. 5. Weibull plots and curve fits of peak-applied-voltage $\left(V_{p k}\right)$ data for type III (no modifications) samples tested with high-voltage electrode type $\mathrm{C}$ (plane).

(pin) electrode. The field enhancement under the point-plane geometry can be estimated using (3)[12] to calculate the peak field, $E_{p k}$, at the tip of the point:

$$
E_{p k}=\frac{2 V}{r_{p} \cdot \ln \left(\frac{4 d}{r_{p}}\right)}
$$

where $V$ is the voltage across the electrodes, $r_{p}$ is the radius of the pin, and $d$ is the interelectrode gap length. For $r_{p}=0.5 \mathrm{~mm}$, $d=10 \mathrm{~mm}$, and $V$ up to $450 \mathrm{kV}$, the estimated peak fields approach $4 \mathrm{MV} / \mathrm{cm}$.

In order to estimate the peak field with collar-plane electrodes, this geometry was modeled using the electrostatic field solver QuickField (Tera Analysis Ltd.). The estimated peak field was $\sim 1 \mathrm{MV} / \mathrm{cm}$, hence the lower $\beta$ values for the BIII geometry when compared with AI.

The Weibull plots of the peak-applied-voltage data for the collar-plane (BIII) and plane-parallel (CIII) electrodes are shown in Figs. 4 and 5, respectively. Comparing Fig. 3 with the collar-plane plots in Fig. 4, it is clear that the probability of breakdown is generally less sensitive to increasing applied voltage for the type B (collar) electrode than for the type A (pin) electrode, evidenced by the shallower gradients of the collarplane plots.

The plane-parallel plots (Fig. 5) for PP, LDPE, Rexolite, and Torlon suggest a similar behavior for these materials. The behavior for UHMWPE is best described by a two-parameter Weibull distribution, and hence there is a clear distinction between the UHMWPE plane-parallel plot and those for the other materials.

\section{B. Voltage at Breakdown}

The calculated values of $\alpha, \beta$, and $\gamma$ for each of the materials and electrode types are listed in Table II for the voltage-atbreakdown data. A three-parameter distribution was fit to these data, with $V$ being replaced by recorded values for $V_{b r}$ in (1) and (2). As in Table I, cases where $\alpha$ represents the characteristic voltage $(\gamma=0)$ have been indicated by an asterisk,
TABLE II

Weibull Distribution PARAmeters For $V_{b r}$

*indicates instances where $\alpha$ is the characteristic voltage, where $F(V)=0.632$

\begin{tabular}{c|c|c|c|c|c}
\hline \hline Geometry & Material & $\begin{array}{c}\text { Scale } \\
\alpha(\mathrm{kV})\end{array}$ & $\begin{array}{c}\text { Shape } \\
\beta\end{array}$ & $\begin{array}{c}\text { Location } \\
\gamma(\mathrm{kV})\end{array}$ & $\begin{array}{c}\alpha+\gamma \\
(\mathrm{kV})\end{array}$ \\
\hline \multirow{3}{*}{$\begin{array}{c}\text { Pin-plane } \\
\text { (AI) }\end{array}$} & LDP & 94 & 1.54 & 155 & 249 \\
$d=10 \mathrm{~mm}$ & UHMWPE & $209^{*}$ & 5.11 & 0 & 209 \\
& Rexolite & $228^{*}$ & 5.11 & 0 & 238 \\
& Torlon & $172^{*}$ & 9.29 & 0 & 228 \\
& PP & $327^{*}$ & 5.31 & 0 & 172 \\
\hline \multirow{4}{*}{ Collar-plane } & LDPE & 162 & 3.00 & 110 & 272 \\
(BIII) & UHMWPE & 49 & 1.38 & 195 & 244 \\
$d=10 \mathrm{~mm}$ & Rexolite & 94 & 1.90 & 175 & 269 \\
& Torlon & $200^{*}$ & 4.15 & 0 & 200 \\
\hline \multirow{3}{*}{ Plane-parallel } & PP & 110 & 1.95 & 115 & 225 \\
(CIII) & LDPE & $217^{*}$ & 4.54 & 0 & 217 \\
$d=4 \mathrm{~mm}$ & UHMWPE & 57 & 1.57 & 98 & 155 \\
& Rexolite & 126 & 3.80 & 75 & 201 \\
& Torlon & 41 & 1.15 & 75 & 116 \\
\hline \multicolumn{5}{r}{}
\end{tabular}

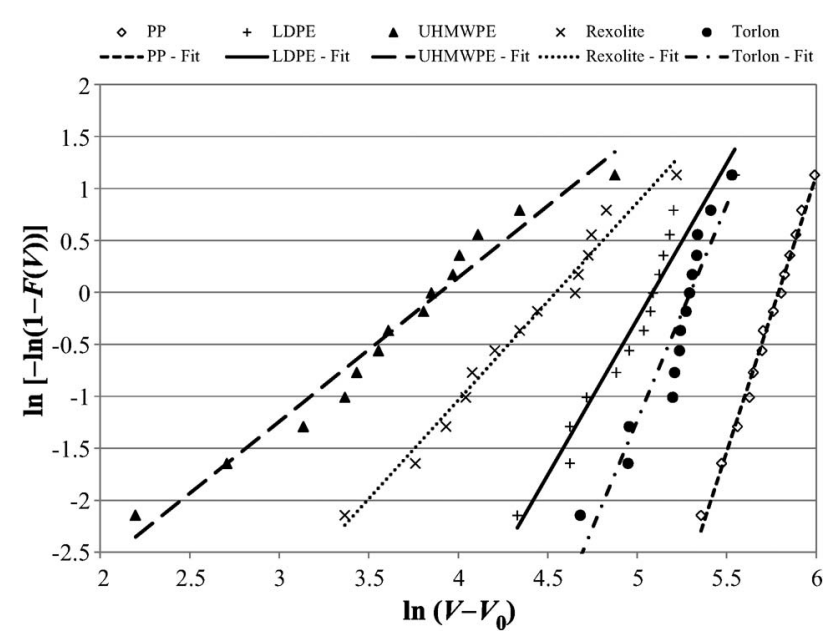

Fig. 6. Weibull plots and curve fits of voltage-at-breakdown $\left(V_{b r}\right)$ data for type III (no modifications) samples tested with high-voltage electrode type B (collar).

and $\alpha+\gamma$ values have been listed in the final column. Again, the materials are generally ranked by $\varepsilon_{r}$ when comparing the characteristic voltage.

For type I samples tested with high-voltage electrode type A (pin), the data for LDPE, UHMWPE, and Rexolite were all best described by a two-parameter Weibull distribution $(\gamma=0)$, and the curves are almost identical. Torlon data were also best described by a two-parameter distribution, although the gradient of the line is twice that of the three aforementioned materials.

For collar-plane geometry (BIII), there is a clear distinction in the behavior of each of the materials, and the Weibull plots are presented in Fig. 6. The location parameter $\gamma$ decreases from $195 \mathrm{kV}$ for UHMWPE to zero for PP and Torlon. The shape parameter $\beta$ increases from 1.38 for UHMWPE to 5.31 for PP.

Under plane-parallel configuration (CIII), the probability of breakdown for Torlon is the least sensitive to increasing voltage $(\beta=1.15)$. PP has the highest $\gamma$ value of $115 \mathrm{kV}$. 
TABLE III

Weibull Distribution PARAMETERS For $t_{b r}$

\begin{tabular}{c|c|c|c}
\hline \hline Geometry & Material & $\begin{array}{c}\text { Scale } \\
\alpha_{t}(\mathrm{~ns})\end{array}$ & $\begin{array}{c}\text { Shape } \\
\beta_{t}\end{array}$ \\
\hline & PP & 463 & 6.13 \\
Pin-plane & LDPE & 617 & 2.10 \\
(AI) & UHMWPE & 434 & 1.57 \\
$d=10 \mathrm{~mm}$ & Rexolite & 538 & 3.61 \\
& Torlon & 407 & 2.83 \\
\hline & PP & 356 & 1.79 \\
Collar-plane & LDPE & 371 & 2.06 \\
(BIII) & UHMWPE & 254 & 2.34 \\
$d=10 \mathrm{~mm}$ & Rexolite & 349 & 2.07 \\
& Torlon & 210 & 4.73 \\
\hline & PP & 422 & 2.67 \\
Plane-parallel & LDPE & 370 & 1.62 \\
(CIII) & UHMWPE & 521 & 2.44 \\
$d=4 \mathrm{~mm}$ & Rexolite & 408 & 4.55 \\
& Torlon & 459 & 2.87 \\
\hline \hline
\end{tabular}

\section{Time to Breakdown}

While the clear distinction in material behavior identified in Fig. 6 is interesting, $V_{b r}$ data were measured on the impulse falling edge. Therefore, time-to-breakdown data were also analyzed to provide a more illuminating analysis of the effect of solid material on the insulation performance of the combined liquid-solid gap. These data were best described by a two-parameter Weibull distribution, where the cumulative probability of failure at time $t$ is defined by (4)[7]:

$$
F(t)=1-\exp \left[-\left(\frac{t}{\alpha_{t}}\right)^{\beta_{t}}\right] .
$$

For this analysis, $t$ was replaced by data for $t_{b r}$ recorded in [1]. The values of $\alpha_{t}$ and $\beta_{t}$ extracted from the $t_{b r}$ data are listed in Table III. The scale parameter, $\alpha_{t}$, can be considered as the characteristic time to breakdown, as $F(t)=0.632$ when $t=\alpha_{t}$.

Of note from this analysis is the behavior under collar-plane geometry, and the Weibull plots for this data are shown in Fig. 7. It is clear that materials with $\varepsilon_{r}$ close to that of the surrounding mineral oil $\left(\varepsilon_{r} \sim 2.2\right)$, that is PP $\left(\varepsilon_{r} \sim 2.25\right)$, LDPE $\left(\varepsilon_{r} \sim\right.$ $2.2)$, and Rexolite $\left(\varepsilon_{r} \sim 2.5\right)$, all show similar performance. The characteristic time to breakdown, $\alpha_{t}$, decreases as the $\varepsilon_{r}$ of the material increases, however, as evidenced by the lower $\alpha_{t}$ values for UHMWPE $\left(\varepsilon_{r} \sim 3.0\right)$ and Torlon $\left(\varepsilon_{r} \sim\right.$ 3.9). The high value of $\beta_{t}$ for Torlon, illustrated by the steep gradient for the Torlon fit in Fig. 7, is indicative of a greater sensitivity to an increase in impulse duration than the other materials.

Analyzing the uniform-field data (geometry CIII), it is evident that the value of $\alpha_{t}$ for UHMWPE is actually longer than that for the other materials of lower $\varepsilon_{r}$, suggesting that another property of UHMWPE results in long times to breakdown. This behavior has been witnessed previously for impulses of longer duration [13], [14], and it is speculated that the ultrahighmolecular-weight additive may be the reason for the longer times to breakdown for UHMWPE [15].

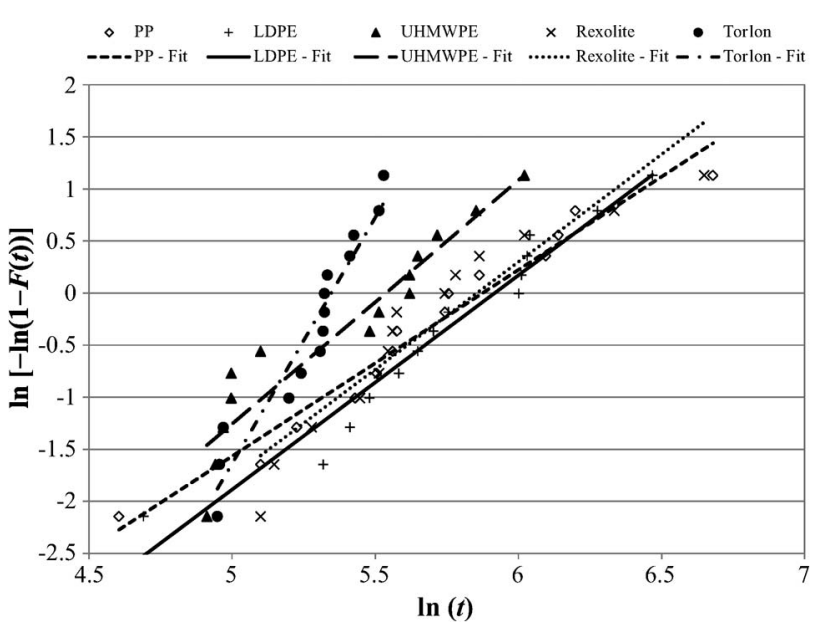

Fig. 7. Weibull plots and curve fits of time-to-breakdown $\left(t_{b r}\right)$ data for type III (no modifications) samples tested with high-voltage electrode type B (collar).

TABLE IV

Average Streamer Propagation Velocities $\left(d / t_{b r}\right)$ IMPLIED BY TIME-TO-BREAKDOWN DATA

\begin{tabular}{|c|c|c|c|c|}
\hline Geometry & Material & $\begin{array}{l}\text { Average } \\
\text { Velocity } \\
(\mathrm{km} / \mathrm{s})\end{array}$ & $\begin{array}{c}\text { Upper } \\
\text { Velocity } \\
(\mathrm{km} / \mathrm{s})\end{array}$ & $\begin{array}{c}\text { Streamer } \\
\text { Mode } \\
{[9]}\end{array}$ \\
\hline \multirow{5}{*}{$\begin{array}{c}\text { Pin/ } \\
\text { No mods. } \\
\text { (AI) }\end{array}$} & $\mathrm{PP}$ & $26 \pm 10$ & 60 & $3^{\text {rd }}$ \\
\hline & LDPE & $25 \pm 17$ & 74 & $3^{\text {rd }}$ \\
\hline & UHMWPE & $38 \pm 26$ & 88 & $3^{\text {rd }}$ \\
\hline & Rexolite & $22 \pm 7$ & 37 & $3^{\text {rd }}$ \\
\hline & Torlon & $30 \pm 8$ & 43 & $3^{\text {rd }}$ \\
\hline \multirow{5}{*}{$\begin{array}{c}\text { Collar/ } \\
\text { No mods. } \\
\text { (BIII) }\end{array}$} & PP & $43 \pm 27$ & 106 & $4^{\text {th }} / 3^{\text {rd }}$ \\
\hline & LDPE & $40 \pm 24$ & 98 & $4^{\text {th }} / 3^{\text {rd }}$ \\
\hline & UHMWPE & $31 \pm 12$ & 49 & $3^{\text {rd }}$ \\
\hline & Rexolite & $53 \pm 23$ & 109 & $4^{\text {th }} / 3^{\text {rd }}$ \\
\hline & Torlon & $40 \pm 19$ & 89 & $3^{\text {rd }}$ \\
\hline \multirow{5}{*}{$\begin{array}{c}\text { Plane/ } \\
\text { No mods. } \\
\text { (CIII) }\end{array}$} & PP & $12 \pm 4$ & 20 & $3^{\text {rd } / 2^{\text {nd }}}$ \\
\hline & LDPE & $18 \pm 11$ & 45 & $3^{\text {rd }} / 2^{\text {nd }}$ \\
\hline & UHMWPE & $13 \pm 14$ & 63 & $3^{\text {rd }} / 2^{\text {nd }}$ \\
\hline & Rexolite & $11 \pm 3$ & 17 & $3^{\mathrm{rd}} / 2^{\text {nd }}$ \\
\hline & & $12 \pm 6$ & 30 & $3^{\text {rd }} / 2^{\text {nd }}$ \\
\hline
\end{tabular}

\section{IMPLIED STREAMER VELOCITIES}

At such short time intervals and high $d V / d t$, a streamer mechanism of breakdown can be assumed to be integral to the breakdown process. While the actual velocity of the streamer that propagates to the point of breakdown cannot be accurately determined without spatial and temporal imaging of the streamer development, an average streamer velocity, estimated by dividing the interelectrode gap length, $d$, by the time to breakdown, $t_{b r}$, can provide a minimum implied velocity of streamer propagation, and a basis for comparison with the results from other studies.

The implied average streamer propagation velocities are summarized in Table IV. The term average velocity has been used to refer to the estimated streamer velocity, averaged over all 15 breakdown events. The \pm values indicate standard deviation. The term upper velocity refers to the estimated velocity yielded by the shortest time to breakdown. The streamer modes have been identified as classified in [9]. 
The velocity of sound in mineral oil has previously been measured as $1.5 \mathrm{~km} / \mathrm{s}$ [16], so streamers generated under the conditions investigated here propagate with supersonic velocity in all cases. The estimated average velocities are in the range of $20-50 \mathrm{~km} / \mathrm{s}$ in nonuniform fields (geometries AI and BIII). The maximum upper velocity exceeds $100 \mathrm{~km} / \mathrm{s}$ for PP and Rexolite in geometry BIII. For uniform-field measurements (geometry CIII), the average velocities are lower, generally of the order of $11-13 \mathrm{~km} / \mathrm{s}$, with the exception of LDPE. The highest upper velocity is greater than $60 \mathrm{~km} / \mathrm{s}$, for UHMWPE.

In nonuniform fields, the upper streamer velocities estimated here are faster than those in the case of open oil gaps without solid spacers [17], where the maximum estimated velocity approached $30 \mathrm{~km} / \mathrm{s}$ for pin-plane geometry, and $20 \mathrm{~km} / \mathrm{s}$ for collar-plane geometry. Under uniform-field conditions, the estimated velocities in open oil gaps [17] were very similar to those shown in Table IV. The electrodes, the mineral oil, the interelectrode gap length, the voltage waveform, and the measurement technique utilized in [17] were the same as for the present paper, and [1].

Streamer velocities up to $300 \mathrm{~km} / \mathrm{s}$ in mineral oil have previously been measured by Lesaint and Massala [18], who found that such fast streamers could be observed through the inclusion of a solid pressboard surface parallel to the electric field, in a similar arrangement to the nonuniform-field geometries utilized in the present study. Lesaint and Massala achieved these measurements in a $10-\mathrm{cm}$ point-plane gap, with a steel point of radius $100 \mu \mathrm{m}$ forming the high-voltage electrode.

\section{Discussion And CONClusion}

Impulse-voltage-breakdown data have been obtained for five dielectric materials considered for use as oil-immersed insulators in high-voltage, pulsed-power machines.

Cumulative failure probability distributions were produced for voltage data obtained in surface-breakdown initiation measurements, following a method similar to the new step-up method [6]. Some data were best described by two-parameter Weibull distributions, suggesting that the breakdown strength will steadily decrease with increasing shot number. For those data with a positive-value location parameter, $\gamma$, this suggests that there is a minimum voltage/field value below which breakdown will not occur, independent of shot number [10]. Analyzing peak-applied-voltage $\left(V_{p k}\right)$ data, the minimum field value is highest for PP for all three tested electrode configurations. The Weibull plots for high-voltage electrode type B (collar) were generally of shallower gradient than for high-voltage electrode type A (pin), suggesting that the probability of breakdown was more sensitive to increases in applied voltage for the pin geometry compared with the collar geometry. Comparing data for the pin-plane and collar-plane geometries in [1], higher applied voltages were required to initiate the first flashover with high-voltage electrode type B (collar), suggesting that the field enhancement provided by the pin was greater than that provided by the collar.

When designing a high-voltage system, the length of the insulator should be chosen using information on the known maximum operating voltage of the system and its temporal behavior, so that the maximum electric-field stress does not exceed a value significantly lower than the mean average applied field threshold for breakdown. This could lead to a reduction in downtime for multimegavolt systems. The values of $\gamma$ obtained here using Weibull statistical analysis provide an estimate of the applied voltage below which breakdown will not occur for a given solid dielectric material, and PP shows the highest values of $\gamma$ for all tested configurations. Only PP consistently reflected a low value of $\beta$, a measure of the sensitivity of the insulation system to an increase in applied voltage, across the different sets of test conditions.

Statistical analysis of time-to-breakdown $\left(t_{b r}\right)$ data, this time utilizing a two-parameter Weibull distribution, revealed that solid materials with $\varepsilon_{r}$ closely matched to that of the surrounding mineral oil generally yielded longer times to breakdown than materials of higher $\varepsilon_{r}$. The longer times to breakdown recorded for UHMWPE are thought to be an effect of the ultrahigh-molecular-weight additive [15], suggesting that this material may be a more effective choice for solid insulation immersed in fluids of higher $\varepsilon_{r}$ such as vegetable or seed oils $\left(\varepsilon_{r} \sim 3.0\right.$ [19]) or ester fluids $\left(\varepsilon_{r} \sim 3.2\right.$ [9]), rather than the mineral oil $\left(\varepsilon_{r} \sim 2.2\right)$ used in the present study.

Similar experimental studies and statistical analysis to the present work, only with an applied voltage wave shape of $1 / 6.5 \mu \mathrm{s}$, can be found in [13], [14]. The similarity of this wave shape to a short-tail lightning impulse means that these results may also be of interest to designers of power systems.

Average streamer propagation velocities, implied by timeto-breakdown data, have also been calculated. Streamers propagate with supersonic velocity for all conditions investigated here, and the maximum estimated velocity exceeds $100 \mathrm{~km} / \mathrm{s}$.

The assumption in calculating streamer velocity using information only on the time to breakdown and the interelectrode gap length is that the statistical time lag will be short at such high electric field and rate of voltage rise, and therefore the major contributor to the time to breakdown will be the formative time lag associated with streamer development and propagation. As discussed in Section IV, spatial and temporal imaging of streamer development would be required in order to directly investigate streamer velocity.

In future work, Laue plots of breakdown time distributions may help to separate the contributions of statistical time lag and formative time lag to the overall time to breakdown.

\section{REFERENCES}

[1] M. P. Wilson, S. J. MacGregor, M. J. Given, I. V. Timoshkin, M. A. Sinclair, K. J. Thomas, and J. M. Lehr, "Surface flashover of oilimmersed dielectric materials in uniform and non-uniform fields," IEEE Trans. Dielectr. Electr. Insul., vol. 16, no. 4, pp. 1028-1036, Aug. 2009.

[2] High-Voltage Test Techniques-Part 1: General Definitions and Test Requirements, Int. Std. IEC 60060-1, Sep. 2010.

[3] R. J. Taylor, "Effect of permittivity matching on the flashover of solid/ liquid interfaces," Proc. IEE, vol. 124, no. 10, pp. 899-904, Oct. 1977.

[4] M. Krins, H. Borsi, and E. Gockenbach, "Impact of carbon particles on the impulse flashover behavior of different solid/liquid interfaces in a nonuniform field," in Proc. Int. Symp. Elect. Insul. Mater., 1998, pp. 363-368.

[5] H. Hirose, "More accurate breakdown voltage estimation for the new step-up test method," IEEE Trans. Dielectr. Electr. Insul., vol. 10, no. 3, pp. 475-482, Jun. 2003. 
[6] H. Hirose, "More accurate breakdown voltage estimation for the new stepup test method in the Weibull model," IEEE Trans. Dielectr. Electr. Insul., vol. 11, no. 3, pp. 418-423, Jun. 2004.

[7] W. Weibull, "Statistical distribution function of wide applicability," Trans. ASME J. Appl. Mech., vol. 18, no. 3, pp. 293-297, 1951.

[8] M. M. Morcos and S. E. Cherukupalli, "Review of statistical testing of solid insulating materials," in Proc. 3rd Int. Conf. Conduct. Breakdown Solid Dielectr., 1989, pp. 574-581.

[9] R. Liu, C. Törnkvist, V. Chandramouli, O. Girlanda, and L. A. A. Pettersson, "Ester fluids as alternative for mineral oil: The difference in streamer velocity and LI breakdown voltage," in Proc. Annu. Rep. CEIDP, 2009, pp. 543-548.

[10] Guide for the Statistical Analysis of Electrical Insulation Breakdown Data, Int. Std. IEC 62539(E): 2007 (IEEE Std. 930-2004), Jul. 2007.

[11] W. W. Dorner (1999). Using Microsoft excel for Weibull analysis. Qual. Dig. [Online]. accessed 14th October 2011. Available: http://www. qualitydigest.com/jan99/html/body_weibull.html

[12] R. Coelho and J. Debeau, "Properties of the tip-plane configuration," J. Phys. D, Appl. Phys., vol. 4, no. 9, pp. 1266-1280, Sep. 1971.

[13] M. P. Wilson, I. V. Timoshkin, M. J. Given, S. J. MacGregor, M. A. Sinclair, K. J. Thomas, and J. M. Lehr, "Effect of applied field and rate of voltage rise on surface breakdown of oil-immersed polymers," IEEE Trans. Dielectr. Electr. Insul., vol. 18, no. 4, pp. 1003-1010, Aug. 2011.

[14] M. P. Wilson, M. J. Given, I. V. Timoshkin, S. J. MacGregor, M. A. Sinclair, K. J. Thomas, and J. M. Lehr, "Impulse-breakdown characteristics of polymers immersed in insulating oil," IEEE Trans. Plasma Sci., vol. 38, no. 10, pp. 2611-2619, Oct. 2010.

[15] M. Kawahigashi, Y. Miyashita, and H. Kato, "Influence of morphology on electrical breakdown strength for polypropylene," in Proc. 3rd Int. Conf. Prop. Appl. Dielectr. Mater., 1991, pp. 1156-1160.

[16] R. Ohyama, A. Watson, and J. S. Chang, "Electrical current conduction and electrohydrodynamically induced fluid flow in an AW type EHD pump," J. Electrost., vol. 53, no. 2, pp. 147-158, Aug. 2001.

[17] M. P. Wilson, I. V. Timoshkin, M. J. Given, S. J. MacGregor, M. A. Sinclair, K. J. Thomas, and J. M. Lehr, "Effect of electrode geometry and rate of voltage rise on streamer propagation in mineral oil," in Proc. IEEE ICDL, 2011, pp. 1-4.

[18] O. Lesaint and G. Massala, "Transition to fast streamers in mineral oil in the presence of insulating solids," in Conf. Rec. IEEE Int. Symp. Elect. Insul., 1996, vol. 2, pp. 737-740.

[19] E. A. Al-Ammar, "Evaluation of seed oils based on statistical breakdown data for their application as insulating fluids in distribution transformers," Eur. J. Sci. Res., vol. 40, no. 1, pp. 15-26, Feb. 2010.

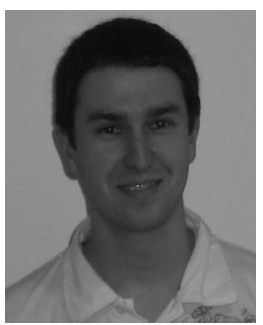

Mark P. Wilson (M'10) was born in Stranraer, Scotland, in 1982. He received the B.Eng. (with honours), M.Phil., and Ph.D. degrees in electronic and electrical engineering from the University of Strathclyde, Glasgow, U.K., in 2004, 2007, and 2011, respectively.

$\mathrm{He}$ is presently working as a Teaching Associate at the University of Strathclyde, where he continues to investigate surface flashover of solids immersed in insulating oil.

Dr. Wilson is a member of the IEEE Nuclear and Plasma Sciences Society, from whom he received a Graduate Scholarship Award in 2011, the IEEE Dielectrics and Electrical Insulation Society, and the IET.

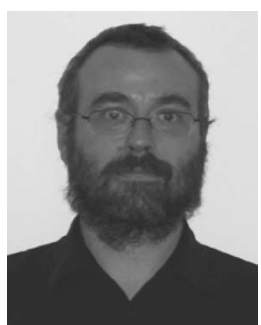

Martin J. Given (M'99-SM'11) received the degree in physics from the University of Sussex, Brighton, U.K., in 1981 and a Ph.D. degree in electronic and electrical engineering from the University of Strathclyde, Glasgow, U.K., in 1996.

He is currently a Senior Lecturer in the Department of Electronic and Electrical Engineering at the University of Strathclyde. His research interests include ageing processes and condition monitoring in solid and liquid insulation systems, high-speed switching, and pulse power applications.

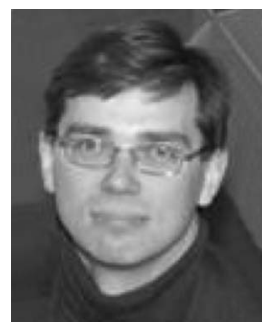

Igor V. Timoshkin (M'07) received the degree in physics from the Moscow State University, Moscow, Russia, in 1992, and the Diploma and the Ph.D. degree from the Imperial College of Science, Technology, and Medicine, London, U.K., in 2001. After graduation from Moscow State University (MSU), he worked as a Researcher at Moscow State AgroEngineering University, and then at the Institute for High Temperatures of Russian Academy of Sciences before moving to Imperial College of Science, Technology, and Medicine (ICSTM) in 1997.

He joined the Department of Electronic and Electrical Engineering of the University of Strathclyde, Glasgow, U.K., in 2001 as an Academic Visitor, where he became a Research Fellow in 2002, a Lecturer in 2006, and a Senior Lecturer in 2011. His research interests include properties of solid and liquid dielectric materials, electronics of plasma discharges in condensed media, practical applications of electrohydraulic and high-power ultrasound pulses, biodielectrics, and effects of electromagnetic fields on biological objects.

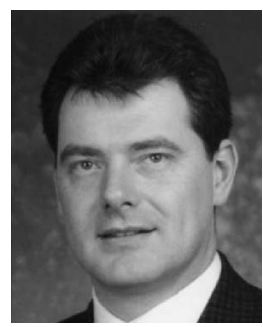

Scott J. MacGregor (M'95) received the B.Sc. and $\mathrm{Ph} . \mathrm{D}$. degrees from the University of Strathclyde, Glasgow, U.K., in 1982 and 1986, respectively.

He is currently with the University of Strathclyde, where he became a Pulsed-Power Research Fellow in 1986, a Lecturer in pulsed-power technology in 1989, a Senior Lecturer in 1994, and a Reader and a Professor of high-voltage engineering in 1999 and 2001, respectively. He has been Dean of Engineering at the University of Strathclyde since January 2010. His research interests include high-voltage pulse generation, high-frequency diagnostics, high-power repetitive switching, highspeed switching, electronic methods for food pasteurization and sterilization, generation of high-power ultrasound (HPU), plasma channel drilling, pulsedplasma cleaning of pipes, and stimulation of oil wells with HPU.

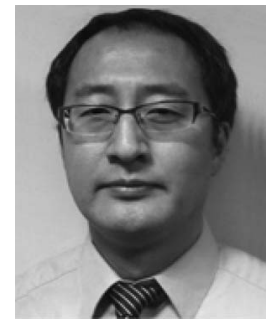

Tao Wang received the B.Eng. and M.Sc. degrees from Northeast China Dianli University (China), in 1993 and 1996, respectively, and the Ph.D. degree from the University of Strathclyde, Glasgow, U.K., in 2005 .

He then joined the Newland Entech as a research fellow developing high efficiency industrial ozone generator. He joined the department of Electronic and Electrical Engineering of University of Strathclyde as a lecturer in 2010. His research interests include non-thermal gas discharges and their applications in gas synthesis, water disinfection and advanced oxidation process in water.

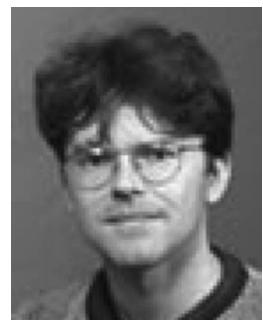

Mark A. Sinclair (M'03) received the B.Sc. degree from the Victoria University of Manchester, U.K., in 1990 and then went to St. Andrews and Strathclyde Universities in Scotland to receive an M.Sc. degree in laser engineering and pulsed-power technology.

In 1992, he joined the Pulsed-Power Group at Atomic Weapons Establishment (AWE). Initially, he worked on the E Minor machine moving on to Mogul E which was optimized for radiography of dense objects. Currently, he is the technical lead and Team Leader for Pulsed Power at AWE running a number of programs to develop fundamental knowledge and skills, to develop new $\mathrm{x}$-ray sources and to use pulsed power for hydrodynamic simulation. 


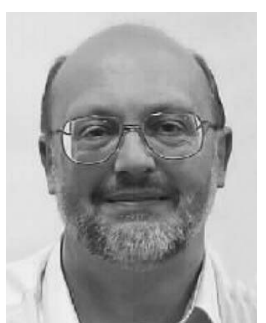

Kenneth J. Thomas (M'05-SM'10) was born in Carmarthen, South Wales, in 1958. He received the B.Sc degree in physics from Imperial College, London, U.K., in 1979. He completed the Ph.D. degree in laser physics, also at Imperial College, in 1983, and joined the pulsed-power group at AWE.

Since then, he has been involved in the design and construction of multimegavolt pulsed-power machines for flash radiography and supporting research, particularly the application of induction voltage adder technology to the next generation of accelerators. He is a C. Phys. and a member of the IET and IoP.

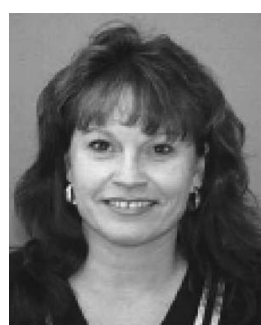

Jane M. Lehr (S'91-M'96-SM'02-F'08) received the B.Eng. degree from the Stevens Institute of Technology, Hoboken, NJ, and the Ph.D. degree in electrophysics from Polytechnic University, New York, NY.

Since 2002, she has been a Member of the Technical Staff with the Exploratory Pulsed-Power Technologies Branch, Sandia National Laboratories, Albuquerque, NM. Prior to joining Sandia National Laboratories, she was with the Directed Energy Directorate, Air Force Research Laboratory.

Dr. Lehr is the Past President of the IEEE Nuclear and Plasma Sciences Society. 